\title{
BMJ Open Postdischarge mortality in children with acute infectious diseases: derivation of postdischarge mortality prediction models
}

\author{
M O Wiens, ${ }^{1}$ E Kumbakumba, ${ }^{2}$ C P Larson, ${ }^{3} \mathrm{~J} \mathrm{M} \mathrm{Ansermino,}{ }^{4} \mathrm{~J}$ Singer,,${ }^{1,5}$ \\ $\mathrm{N}$ Kissoon, ${ }^{6} \mathrm{H}$ Wong, ${ }^{1}$ A Ndamira, ${ }^{2} \mathrm{~J}$ Kabakyenga, ${ }^{7} \mathrm{~J}$ Kiwanuka, ${ }^{2} \mathrm{G}$ Zhou $^{8}$
}

To cite: Wiens M0, Kumbakumba $\mathrm{E}$, Larson $\mathrm{CP}$, et al. Postdischarge mortality in children with acute infectious diseases: derivation of postdischarge mortality prediction models. BMJ Open 2015;5:e009449. doi:10.1136/bmjopen-2015009449

- Prepublication history for this paper is available online. To view these files please visit the journal online (http://dx.doi.org/10.1136/ bmjopen-2015-009449).

Received 21 July 2015 Accepted 16 October 2015

CrossMark

For numbered affiliations see end of article.

Correspondence to Dr Matthew Wiens; mowiens@outlook.com

\section{ABSTRACT}

Objectives: To derive a model of paediatric postdischarge mortality following acute infectious illness.

Design: Prospective cohort study.

Setting: 2 hospitals in South-western Uganda.

Participants: 1307 children of 6 months to 5 years of age were admitted with a proven or suspected infection. 1242 children were discharged alive and followed up 6 months following discharge. The 6-month follow-up rate was $98.3 \%$.

Interventions: None.

Primary and secondary outcome measures: The primary outcome was postdischarge mortality within 6 months following the initial hospital discharge.

Results: 64 children died during admission (5.0\%) and 61 died within 6 months of discharge $(4.9 \%)$. Of those who died following discharge, $31(51 \%)$ occurred within the first 30 days. The final adjusted model for the prediction of postdischarge mortality included the variables mid-upper arm circumference (OR $0.95,95 \% \mathrm{Cl} 0.94$ to 0.97 , per $1 \mathrm{~mm}$ increase), time since last hospitalisation (OR $0.76,95 \% \mathrm{Cl} 0.61$ to 0.93 , for each increased period of no hospitalisation), oxygen saturation (OR $0.96,95 \% \mathrm{Cl}$ 0.93 to 0.99 , per $1 \%$ increase), abnormal Blantyre Coma Scale score (OR 2.39, 95\% Cl 1.18 to 4.83 ), and HIV-positive status (OR 2.98, $95 \% \mathrm{Cl} 1.36$ to 6.53). This model produced a receiver operating characteristic curve with an area under the curve of 0.82 . With sensitivity of $80 \%$, our model had a specificity of $66 \%$. Approximately $35 \%$ of children would be identified as high risk $(11.1 \%$ mortality risk) and the remaining would be classified as low risk (1.4\% mortality risk), in a similar cohort.

Conclusions: Mortality following discharge is a poorly recognised contributor to child mortality. Identification of at-risk children is critical in developing postdischarge interventions. A simple prediction tool that uses 5 easily collected variables can be used to identify children at high risk of death after discharge. Improved discharge planning and care could be provided for high-risk children.

\section{Strengths and limitations of this study}

- The primary strengths of this study are prospective and rigourous data collection, and nearcomplete follow-up.

- Further strengths include the derivation of multiple similar models to allow prediction in circumstances where not all variables may be available.

- Regression models can easily be incorporated into a mobile health-based tool for simple and rapid prediction by health workers.

- The primary limitations of this study are relatively few outcomes, and lack of external validity. Despite few outcomes, our models performed quite well.

- These limitations highlight the need for further research on this important but neglected topic.

- The identification of high risk does not imply that risk can be reduced. Further work is needed on the development of postdischarge interventions to reduce this burden.

\section{BACKGROUND}

Acute infectious diseases continue to be the most important contributor to the 6 million children younger than 5 years who die every year, particularly in Africa. ${ }^{1}$ It is widely accepted that as a global community we have fallen short in reducing under-5 mortality, as demonstrated by the fact that most developing countries, especially those in sub-Saharan Africa will not achieve the fourth millennium development goal of a two-thirds reduction in child mortality. ${ }^{2}$ An important but neglected contributor to infectious disease-related mortality is the vulnerable period following hospital discharge.

A recent systematic review of paediatric studies assessing postdischarge mortality in resource-poor countries found that postdischarge mortality often exceeds in-hospital 
mortality. ${ }^{3}$ Thus, attention to at-risk populations post discharge is sorely needed. However, while several factors were consistently found to be associated with mortality following discharge, including malnutrition, HIV and severe pneumonia, easy identification is essential in order to develop targeted postdischarge interventions. Ideally, the unacceptably high risk of morbidity and mortality following discharge suggests that all children should be afforded follow-up care. However, significant resource constraints in the countries most affected by this issue preclude any significant intervention for all discharged children. Therefore, the ability to quickly and effectively identify at-risk children would be an invaluable step towards the implementation of life-saving postdischarge interventions. An important and easily identified dichotomy among hospital admissions are infectious diseases and non-infectious disease-related admissions such as trauma, cancer and congenital diseases. Although further divisions based on aetiology of infection or an underlying risk factor, such as malnutrition or HIV status, may be an attractive approach in risk stratification, significant difficulties in disease definitions and often overlapping risks makes this approach very difficult. The development of a robust yet simple risk-scoring algorithm could significantly advance a systematic and evidence-based approach in postdischarge care.

The purpose of this study was to derive simple prediction models that could efficiently stratify children according to postdischarge mortality risk.

\section{METHODS}

Population

Mbarara, a city of approximately 195000 , is the largest city in the South-western region of Uganda. This study was conducted at two hospitals in Mbarara. The Mbarara Regional Referral Hospital (MRRH) is the main referral hospital in South-western Uganda. It is a public hospital funded by the Uganda Ministry of Health. MRRH is associated with the Mbarara University of Science and Technology, and is a primary training site for its healthcare graduates. The paediatric ward admits approximately 5000 patients per year. The Holy Innocents Children's Hospital (HICH) is a faith-based children's hospital offering subsidised fee-for-service for outpatient and in-patient care in Mbarara. The HICH admits approximately 2500 patients per year.

This was a prospective observational study conducted between March 2012 and December 2013. This study was approved by the institutional review boards at the University of British Columbia (Canada) and the Mbarara University of Science and Technology (Uganda), as well as the Uganda National Council for Science and Technology and Office of the President. This study was voluntary and written informed consent was provided by a parent or guardian of all children who were enrolled.
Eligibility

All children aged 6 months to 5 years who were admitted with a proven or suspected infection were eligible for enrolment. The upper age limit was chosen to coincide with the under- 5 target group of the millennium development goals. The lower age limit was chosen for logistic (census enrolment with limited research staff) and statistical considerations (group homogeneity). Participants already enrolled in the study were not eligible to be enrolled during subsequent admissions.

\section{Study procedure}

Following enrolment, a research nurse obtained and recorded clinical signs including a $1 \mathrm{~min}$ respiratory rate, blood pressure (automated), axillary temperature, Blantyre Coma Scale (BCS) score, and by using the Phone Oximeter, ${ }^{4}$ the $1 \mathrm{~min}$ photoplethysmogram (PPG), blood oxygen saturation $\left(\mathrm{SpO}_{2}\right)$ and heart rate. Anthropometric data (height, weight, mid-upper arm circumference (MUAC)) were also measured and recorded. Age-dependent demographic variables collected at enrolment were converted to age-corrected z-scores according to the WHO Child Growth Standards. ${ }^{5}$ The age-corrected heart rate and respiratory rate z-scores were obtained by standardising the raw measurements using the median and SD values provided by Fleming et al. ${ }^{6}$ The age-corrected z-scores for systolic blood pressure were calculated using participants' height, according to the procedures previously described. ${ }^{7}$

A blood sample was taken for measurement of haemoglobin, HIV and a malaria blood smear (microscopy). HIV status was determined using the national rapid diagnostic test serial algorithm. ${ }^{8}$ All positive tests on the Determine Antibody Test were confirmed by a separate test (UniGold). Children under 12 months of age with a positive test were confirmed using PCR. Haemoglobin was measured on a Beckman Coulter Ac.T Hematology Analyzer.

An interview was conducted with the participant's parent/guardian and information about previous admissions, distance from health facility, transportation costs, bed-net use, maternal education, maternal age, maternal HIV status, history of sibling deaths and drinking water safety were elicited. Participants received routine care during their hospital stay and were discharged at the discretion of the treating medical team. The discharge status of all enrolled participants was recorded as death, referral, discharged alive, and discharged against medical advice. The diagnoses made by the medical team were also recorded. On discharge, families with active telephone lines were contacted at months 2 and 4 to determine the vital status of the child. Families with no telephone access received in-person follow-up by a field officer. At approximately 6 months following discharge, all participants received in-person follow-up. In addition to postdischarge vital status, health seeking and 
rehospitalisations since the initial discharge were also recorded.

Study data were collected and managed using REDCap electronic data capture tools hosted at the Child and Family Research Institute, Vancouver, Canada. ${ }^{9}$ REDCap (Research Electronic Data Capture) is a secure, web-based application designed to support data capture for research studies and provides: (1) an intuitive interface for validated data entry; (2) audit trails for tracking data manipulation and export procedures; (3) automated export procedures for seamless data downloads to common statistical packages and (4) procedures for importing data from external sources.

Candidate predictor variables were derived using a two-round modified Delphi approach. Briefly, 23 experts in relevant disciplines were solicited to complete an online survey and provide feedback on an initial list of proposed predictors. Predictors were evaluated on considerations of utility as predictors, availability, cost and resource-related applicability. Experts were asked to provide additional potential variables which were then evaluated during a second round of surveys. Data was evaluated by the research team and a final list of candidate predictor variables for modelling was then determined. ${ }^{10}$

\section{Outcomes}

The primary outcome was postdischarge mortality at any time during the 6-month postdischarge period.

\section{Sample size}

For the derivation of prediction models, standard calculations of sample size do not apply since these calculations do not account for the model development process (ie, selection of variables and the optimisation to achieve specified sensitivity and specificity cut-offs). For this study, we determined the sample size needed to validate the derived model and plan to use an equal number of patients for the derivation phase. For the validation study, assuming that the derived model achieves a sensitivity of $85 \%$ with at least $50 \%$ specificity, 100 events, corresponding to a total sample of approximately 1000 live-discharges (assuming a postdischarge mortality rate of $10 \%$ ), would be needed to obtain $80 \%$ power for ensuring that the lower $95 \%$ confidence limit on sensitivity will be at least $75 \%$. Since resources are scarce, a higher sensitivity at the expense of specificity would further limit practical application of such a model. An interim analysis of the study showed that the postdischarge mortality rate would likely not exceed $5 \%$ and enrolment was stopped when 1307 participants were enrolled.

\section{Statistical analysis}

All variables were assessed using univariate logistic regression to determine their level of association with the primary outcome. Continuous variables were assessed for model fit using the Hosmer-Lemeshow test. ${ }^{11}$ Missing data was imputed by the method of multivariate imputation using chained equations. ${ }^{12}$ Following univariate analysis, candidate models were generated using a stepwise selection procedure minimising Akaike's Information Criterion (AIC). This method is considered asymptotically equivalent to cross-validation and bootstrapping. ${ }^{13}{ }^{14}$ All models generated in this sequence having AIC values within $10 \%$ of the lowest value were considered as reasonable candidates. The final selection of a model was judged on model parsimony (the simpler the better), availability of the predictors (with respect to minimal resources and cost), and the attained sensitivity (with at least $50 \%$ specificity). All analyses were conducted using SAS V.9.3 (Carey, North Carolina, USA) and R 3.1.3 (Vienna, Austria; http:// www.R-project.org). Additional models were created using the above process but with the absence of key variables used in deriving the primary model, including a model not including any variables likely to change over the course of admission. This was done to increase application in a variety of settings were certain variables may not be available.

\section{RESULTS}

During the period of study, 1822 participants were screened for eligibility, of which 516 (28\%) were excluded. Reasons for exclusion included isolated malnutrition $(\mathrm{n}=192)$, readmission of previously enrolled participant $(n=51)$, refusal of consent $(n=22)$, cardiac disease $(n=19)$, poisoning/drug reaction $(n=19)$ and cancer $(n=12)$ as well as a plethora of other noninfectious admissions $(n=165)$. In total, 1307 participants admitted with a presumed or proven infection were enrolled at the time of their admission. During the course of admission, $64(5.1 \%)$ participants died, and $1242(94.9 \%)$ were discharged alive (figure 1). Among the children discharged, $54 \%$ were male, and the median age was 18.1 months (IQR 10.8-34.6). Pneumonia, malaria and gastroenteritis were the most common clinical discharge diagnoses and were present in $31 \%, 50 \%$, and $8 \%$ of discharged participants, respectively. According to anthropometric variables collected at admission, $30 \%$ of participants were considered underweight (weight for age z-score $<-2$ ), $35 \%$ were considered wasted (weight for height/length z-score < -2 ), and 29\% were considered stunted (height/length for age $z$-score $<-2$ ) (table 1). Missing observations were minimal (table 2).

\section{Postdischarge mortality}

The rate of successful follow-up during the postdischarge period was $98.3 \%$, with only six participants receiving no follow-up during this period. Overall, 61 $(4.9 \%)$ children died following discharge. Of those who died, the median time to death was 30 days (IQR 7-81). Of the 61 deaths, $41(67 \%)$ occurred outside of a hospital and $20(33 \%)$ occurred during a hospital 
Figure 1 Consort diagram of study flow.

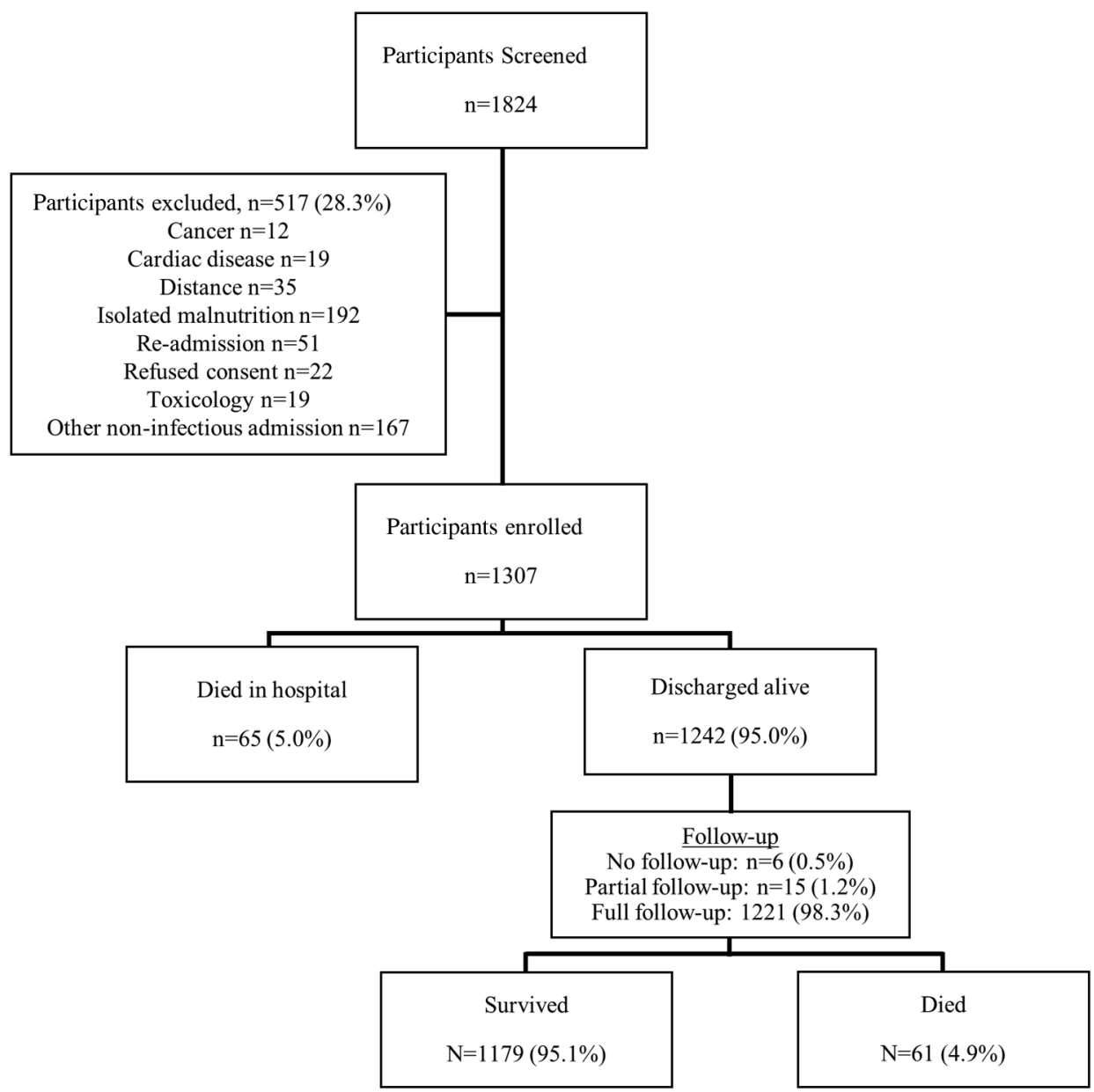

readmission. Thirty variables were tested for univariate associations with postdischarge mortality (table 2). MUAC was the variable with the highest area under the receiver operating characteristic (ROC) curve, 0.76 (95\% CI 0.70 to 0.83$)$, and was highly significant $(\mathrm{p}<0.0001)$. Other anthropometric variables, including weight for age $z$-score, length/height for age $z$-score, and weight for length/height z-score were also highly associated with postdischarge mortality but had much lower areas under the ROC curve. Oxygen saturation was the most predictive of the non-anthropometric variables, with an area under the ROC curve of $0.65(95 \%$ CI 0.57 to 0.73 ), followed by age and parasitaemia with areas under the ROC curve of 0.64 (95\% CI 0.56 to 0.70 ) and 0.60 (95\% CI 0.55 to 0.65$)$, respectively. Other variables achieving statistical significance, but showing lower areas under the ROC curve, included systolic blood pressure, axillary temperature, HIV status, abnormal BCS (yes vs no), duration of illness prior to admission $>7$ days and time since last hospitalisation (analysed as continuous variable and ordered as $<7$ days, 7 to 30 days, 30 days to 1 year, $>1$ year, and never). Haemoglobin level, history of sibling deaths, maternal HIV status, maternal education and distance from admitting health facility were not associated with postdischarge mortality in the univariate analysis.

\section{Multivariate prediction models}

One primary model and three alternate models of equal sensitivity were developed for the prediction of 6-month postdischarge mortality (table 3 ). Two alternate models were developed while systematically excluding oxygen saturation, and HIV status, respectively, since these may not be routinely available in all clinical settings. A fourth model was developed excluding variables most likely to change over the course of admission (ie, clinical variables), giving the model utility for variables collected at any time throughout the hospital stay. The primary model included MUAC in $\mathrm{mm}$, oxygen saturation $\left(\mathrm{SpO}_{2}\right)$ at admission (per cent), time since previous hospitalisation, the presence of abnormal BCS score at admission, and HIV status. The area under the ROC curve was $0.82(95 \%$ CI 0.76 to 0.87 ) (figure 2$)$. The model, at a cut-off of $>80 \%$ sensitivity, had a final sensitivity of $82 \%(95 \%$ CI $0.75 \%$ to $0.87 \%)$ and a specificity of $66 \%(95 \%$ CI $64 \%$ to $69 \%)$. In a population similar to this model derivation cohort, we would expect the positive predictive value to be $11.1 \%$ and the negative predictive value to be $98.6 \%$ (table 4 ). The final model equation for the primary model was: $\operatorname{logit}(p)=7.71+$ $(-0.041 ; \mathrm{MUAC})+\left(-0.041 ; \mathrm{SpO}_{2}\right)+(-0.28 ;$ time period since last hospitalisation $)+(1.09$; HIV positive $)+$ $(0.87 ; \mathrm{BCS}<5)$. 
Table 1 General characteristics of discharged participants $(\mathrm{N}=1242)$

\section{Characteristic}

Frequency (\%)

Age, months

$<12 \quad 378(30)$

$12-24 \mathrm{~m}$

24-36 m

$36-48 \mathrm{~m}$

$>48 \mathrm{~m}$

Male sex

Length of stay, days

$<3$

3-5

$6-10$

$>10$

Discharge AMA

Diagnoses

Pneumonia

Clinical malaria

Parasitaemia

Gastroenteritis

SSTI

Meningitis

Tuberculosis

Measles

Comorbidities

HIV

Sickle cell

Tuberculosis

Admission anthropometric characteristics

Underweight (WAZ<-2)

Severe underweight $(W A Z<-3)$

Wasting $(\mathrm{WHZ}<-2)$

Severe wasting $(\mathrm{WHZ}<-3)$

Stunting $(H A Z<-2)$

Severe stunting $(\mathrm{HAZ}<-3)$

$M U A C<125 \mathrm{~mm}$

MUAC $<115 \mathrm{~mm}$

AMA, against medical advice; HAZ, height/length for age z-score; MUAC, mid-upper arm circumference; SSTI, skin and soft tissue infection; WAZ, weight for age $\mathrm{Z}$-score; WHZ, weight for height/

length $z$-score.

Model 2 excluded oxygen saturation (table 3). The final model included MUAC, time since last hospitalisation, HIV status and the presence of an abnormal BCS score. The area under the ROC curve was $0.81(95 \%$ CI 0.75 to 0.87$)$. This model had a sensitivity of $80 \%(95 \%$ CI $70 \%$ to $90 \%)$ and specificity of $68 \%(95 \%$ CI $65 \%$ to $70 \%$ ), and would generate a positive and negative predictive value of $11.3 \%$ and $98.5 \%$, respectively, in a population similar to the derivation cohort.

The third model excluded HIV status (table 3). This model had a final area under the ROC curve of 0.80 (95\% CI 0.74 to 0.86 ), and a sensitivity of $80 \%$ (95\% CI $70 \%$ to $90 \%$ ) and specificity of $63 \%$ (95\% CI $60 \%$ to $66 \%)$. The positive and negative predictive values were $10.2 \%$ and $98.4 \%$, respectively.

The final model excluded all time-changing clinical parameters (eg, vital signs, $\mathrm{SpO}_{2}$, coma score, etc) so as to be applicable to data collected at any time during admission, including discharge. This model contained only three variables, MUAC, HIV status and the most recent hospitalisation. This model achieved good performance characteristics including an area under the curve (AUC) of 0.80 (95\% CI 0.73 to 0.86 ). The sensitivity was $82 \%(95 \%$ CI $72 \%$ to $92 \%)$ and the specificity was $61 \%(95 \%$ CI $59 \%$ to $64 \%)$, and the positive and negative predictive values were $9.9 \%$ and $98.5 \%$, respectively.

\section{DISCUSSION}

This study represents the first systematic approach to the development of a simple risk-scoring algorithm for postdischarge mortality following admission for an acute infectious illness using prospectively collected data. The variables used in these models are easy to collect and include MUAC, oxygen saturation, BCS score, time since last hospitalisation, and HIV status. Four prediction models were developed to ensure its effective application in a variety of clinical circumstances. All four models had very similar performance characteristics with the most parsimonious model including only MUAC, HIV status and time since last hospitalisation, with only marginally lower AUC than the full model with five variables. The models which were developed use only variables collected at admission and can, therefore, easily be incorporated into the discharge planning process during the hospital stay. Using these models, the identification of at-risk children would ensure that most children likely to die in the postdischarge period (about $80 \%$ ) would be identified. These children have an average mortality risk of approximately over $10 \%$, justifying the exploration of potentially life-saving interventions. Interventions found to be effective could likely be brought to scale without inordinately burdening the already stressed healthcare systems.

The development and implementation of predictive models into routine clinical care is not common in resource-poor countries. The high prevalence of overlapping diseases (such as pneumonia, malaria and malnutrition), and the difficulty in creating reliable diagnostic algorithms to identify eligible populations cause significant difficulty in the application of disease-specific models. To create models with uptake potential, these would need to be linked with existing clinical practices and resources, and would also require a shift in how infectious illness is viewed-not as an episodic disease but as a continuum beyond the acute episode. The Integrated Management of Childhood Illness (IMCI), while not a predictive tool per se, is an algorithm-based approach for the diagnosis and management of acute infectious illnesses. ${ }^{15}$ IMCI has seen significant uptake in many countries throughout sub-Saharan Africa, and has provided a systematic approach to the care of children within health facilities. More importantly, it has been shown to improve care in the regions where it has 
Table 2 Univariate analysis of potential predictor variables

\begin{tabular}{|c|c|c|c|c|}
\hline Variable & $\begin{array}{l}\text { Missing } \\
\text { observation }\end{array}$ & OR $(95 \% \mathrm{Cl})$ & AUC (95\% CI) & p Value \\
\hline Male sex & 0 & $0.90(0.54$ to 1.51$)$ & $0.51(0.45$ to 0.58$)$ & 0.700 \\
\hline Age (months) & 3 & 0.97 (0.97 to 0.97$)$ & 0.64 (0.56 to 0.70$)$ & 0.003 \\
\hline MUAC (mm) & 14 & 0.97 (0.96 to 0.98$)$ & $0.76(0.70$ to 0.83$)$ & $<0.001$ \\
\hline Weight for age z-score & 5 & $0.66(0.57$ to 0.76$)$ & $0.68(0.60$ to 0.76$)$ & $<0.001$ \\
\hline Weight for length/height z-score & 15 & $0.81(0.72$ to 0.91$)$ & $0.62(0.55$ to 0.70$)$ & $<0.001$ \\
\hline Length/height for age z-score & 16 & 0.79 (0.70 to 0.89$)$ & $0.63(0.56$ to 0.71$)$ & $<0.001$ \\
\hline HR for age z-score & 3 & $0.86(0.74$ to 0.99$)$ & 0.61 (0.53 to 0.69$)$ & 0.036 \\
\hline HR (raw) & 0 & $1.00(0.99$ to 1.01$)$ & 0.53 (0.47 to 0.62$)$ & 0.728 \\
\hline RR for age z-score & 3 & 0.99 (0.92 to 1.06$)$ & $0.53(0.45$ to 0.60$)$ & 0.747 \\
\hline $\mathrm{RR}(\mathrm{raw})$ & 0 & 1.01 (1.00 to 1.03$)$ & 0.57 (0.50 to 0.63$)$ & 0.100 \\
\hline SBP z-score & 21 & 0.94 (0.79 to 1.12$)$ & $0.50(0.45$ to 0.61$)$ & 0.526 \\
\hline SBP (raw) & 6 & 0.98 (0.96 to 1.00$)$ & $0.58(0.50$ to 0.66$)$ & 0.018 \\
\hline DBP (raw) & 6 & 0.99 (0.97 to 1.01$)$ & 0.55 (0.50 to 0.65$)$ & 0.255 \\
\hline Temperature (transformed) & 0 & $1.02(0.90$ to 1.16$)$ & 0.51 (0.45 to 0.57$)$ & 0.789 \\
\hline Temperature (raw) & 0 & 0.76 (0.62 to 0.93$)$ & $0.58(0.50$ to 0.65$)$ & 0.007 \\
\hline $\mathrm{SpO}_{2}$ (raw) & 13 & 0.94 (0.92 to 0.96$)$ & 0.65 (0.57 to 0.73$)$ & $<0.001$ \\
\hline $\mathrm{SpO}_{2}$ (transformed) & 13 & 1.04 (1.02 to 1.05$)$ & 0.65 (0.57 to 0.73$)$ & $<0.001$ \\
\hline HIV positive (vs negative) & 25 & $5.21(2.55$ to 10.65$)$ & 0.57 (0.52 to 0.62$)$ & $<0.001$ \\
\hline Haemoglobin $(\mathrm{g} / \mathrm{dL})$ & 10 & $0.95(0.87$ to 1.03$)$ & 0.56 (0.49 to 0.63$)$ & 0.227 \\
\hline Blantyre Coma Scale <5 (vs 5) & 0 & 2.40 (1.27 to 4.57$)$ & 0.56 (0.50 to 0.61$)$ & 0.007 \\
\hline Positive blood smear (vs negative) & 11 & $0.33(0.16$ to 0.68$)$ & 0.60 (0.55 to 0.65$)$ & 0.002 \\
\hline IIIness $>7$ days prior to admission & 1 & 0.50 (0.30 to 0.83$)$ & $0.58(0.52$ to 0.65$)$ & 0.008 \\
\hline Time since last hospitalisation* & 3 & 0.75 (0.62 to 0.90$)$ & 0.59 (0.52 to 0.67$)$ & 0.003 \\
\hline Sibling deaths & 0 & $1.54(0.89$ to 2.65$)$ & 0.55 (0.48 to 0.61$)$ & 0.121 \\
\hline Number of children in family & 2 & $1.02(0.92$ to 1.13$)$ & $0.50(0.43$ to 0.58$)$ & 0.750 \\
\hline Boil all drinking water & 0 & $0.82(0.47$ to 1.42$)$ & $0.52(0.46$ to 0.58$)$ & 0.471 \\
\hline Maternal age (years) & 0 & $1.00(0.97$ to 1.04$)$ & $0.52(0.41$ to 0.57$)$ & 0.892 \\
\hline \multicolumn{5}{|l|}{ Maternal HIV (ref: negative) } \\
\hline HIV positive, $\mathrm{n}=142$ & 0 & $1.79(0.87$ to 3.67$)$ & \multirow[t]{2}{*}{$0.54(0.48$ to 0.61$)$} & 0.113 \\
\hline HIV status unknown, $\mathrm{n}=220$ & 0 & 1.27 (0.64 to 2.52$)$ & & 0.499 \\
\hline \multicolumn{5}{|l|}{ Maternal education (ref: < primary 3) } \\
\hline Primary $3-7, n=630$ & 0 & $1.18(0.62$ to 2.23$)$ & \multirow[t]{3}{*}{0.54 (0.50 to 0.63$)$} & 0.619 \\
\hline Some secondary, $\mathrm{n}=269$ & 0 & $0.72(0.31$ to 1.70$)$ & & 0.457 \\
\hline Postsecondary, $n=93$ & 0 & $1.18(0.41$ to 3.36$)$ & & 0.762 \\
\hline \multicolumn{5}{|l|}{ Bed-net use (ref: never) } \\
\hline Sometimes & 0 & 1.00 (0.48 to 2.09$)$ & \multirow[t]{2}{*}{$0.52(0.45$ to 0.59$)$} & 0.996 \\
\hline Always & 0 & 0.85 (0.46 to 1.58$)$ & & 0.612 \\
\hline \multicolumn{5}{|l|}{ Distance from hospital (ref: <30 min) } \\
\hline $30-60$ & 0 & $0.71(0.31$ to 1.64$)$ & \multirow[t]{2}{*}{0.56 (0.49 to 0.62$)$} & 0.421 \\
\hline$>60$ & 0 & $1.30(0.70$ to 2.41$)$ & & 0.401 \\
\hline
\end{tabular}

*Ordered as $<7$ days, 7 to 30 days, 30 days to 1 year, $>1$ year and never (analysed as continuous and coded and $1-5$, respectively).

AUC, area under the curve; DBP, diastolic blood pressure; HR, heart rate; MUAC, mid-upper arm circumference; RR, respiratory rate; SBP, systolic blood pressure.

been implemented. ${ }^{16}$ However, the IMCI does not address the important issue of postdischarge vulnerability and therefore, fails to provide any guidance beyond the period of acute illness in the hospital, even though the postdischarge period will claim as many lives as the acute hospital period. The integration of a postdischarge risk score into IMCI could begin to address this need.

This study is participant to several limitations. A primary limitation of this study is the relatively low number of outcomes observed. Although our initial sample size estimates were to observe 100 outcomes, we only observed 61 . Our comprehensive follow-up of participants ensured that missed outcomes are unlikely.
Further, the performance of our model was good, with the lower limits of the calculated 95\% CIs for AUC, sensitivity and specificity remaining in an acceptable range. A further limitation is the lack of external validity. While our research sites represented the typical East African context, further research is required to ensure the validity of these models elsewhere, especially in areas with significant differences in the distribution of important diseases such as malaria, diarrhoea and pneumonia, and malnutrition. A limitation to application of the prediction models developed is that the risk score is based on a regression equation and cannot be easily computed without the assistance of a computer or similar device. 
Table 3 Models developed for prediction of 6-month postdischarge mortality

\begin{tabular}{|c|c|c|c|}
\hline Variable & Regression estimate & p Value & OR $(95 \% \mathrm{Cl})$ \\
\hline \multicolumn{4}{|c|}{ Model 1—primary model, intercept $=7.7172$} \\
\hline MUAC & -0.0462 & $<0.0001$ & $0.95(0.94$ to 0.97 \\
\hline $\mathrm{SpO}_{2}$ & -0.0411 & 0.0029 & 0.96 (0.93 to 0.99 \\
\hline Time since last hospitalisation & -0.2775 & 0.0085 & $0.76(0.62$ to 0.93 \\
\hline HIV positive & 1.0915 & 0.0064 & $2.98(1.36$ to 6.53 \\
\hline Abnormal BCS score & 0.8723 & 0.0150 & 2.39 (1.18 to 4.83 \\
\hline \multicolumn{4}{|c|}{ Model 2-model without $\mathrm{SpO}_{2}$, intercept $=4.4538$} \\
\hline MUAC & -0.0505 & $<0.0001$ & 0.95 (0.94 to 9.97 \\
\hline Time since last hospitalisation & -0.2503 & 0.0153 & $0.78(0.64$ to 0.95 \\
\hline HIV positive & 1.0902 & 0.0061 & $2.98(1.37$ to 6.48 \\
\hline Abnormal BCS score & 1.0664 & 0.0022 & $2.91(1.47$ to 5.75 \\
\hline \multicolumn{4}{|c|}{ Model 4-model without HIV, intercept $=8.2813$} \\
\hline MUAC & -0.0492 & $<0.0001$ & $0.95(0.94$ to 0.97 \\
\hline $\mathrm{SpO}_{2}$ & -0.0412 & 0.0027 & $0.96(0.93$ to 0.99 \\
\hline Time since last hospitalisation & -0.2870 & 0.0058 & $0.75(0.61$ to 0.92 \\
\hline Abnormal BCS score & 0.8040 & 0.0248 & $2.23(1.11$ to 4.51$)$ \\
\hline \multicolumn{4}{|c|}{ Model $4-$ model without clinical variables, intercept $=4.4511$} \\
\hline MUAC & -0.0492 & $<0.0001$ & $0.95(0.94$ to 0.97 \\
\hline HIV positive & 1.0143 & 0.0108 & $2.76(1.26$ to 6.01$)$ \\
\hline Time since last hospitalisation & -0.2458 & 0.0164 & $0.78(0.64$ to 0.96 \\
\hline
\end{tabular}

However, with the increasing prevalence of mobile phones in developing countries, health interventions are increasingly focused on utilising the computational power of mobile phones to implement life-saving technology. Several important health interventions use mobile technology to improve care. ${ }^{17-19}$

It is clear that malnutrition plays a major role in postdischarge mortality. MUAC provided a significant proportion of the predictive power in our models by providing an AUC of 0.76 , only $7 \%$ lower than the final full model. No models meeting our prespecified criteria could be developed without the use of any anthropomorphic measure. The importance of malnutrition has also been clearly demonstrated in other studies of postdischarge mortality. ${ }^{20-22}$ Although first described over 50 years ago, environmental enteropathy (also called tropical enteropathy or environmental enteric dysfunction) has received significant attention in recent years. It has been suggested that changes in the gut microbiome and the small intestinal wall (flattened villi,
Figure 2 Performance of the primary prediction model derived with data from admission (AUC, area under the curve; NPV, negative predictive value; PPV, positive predictive value; $\mathrm{ROC}$, receiver operating characteristic; Sens, sensitivity; Spec, specificity).

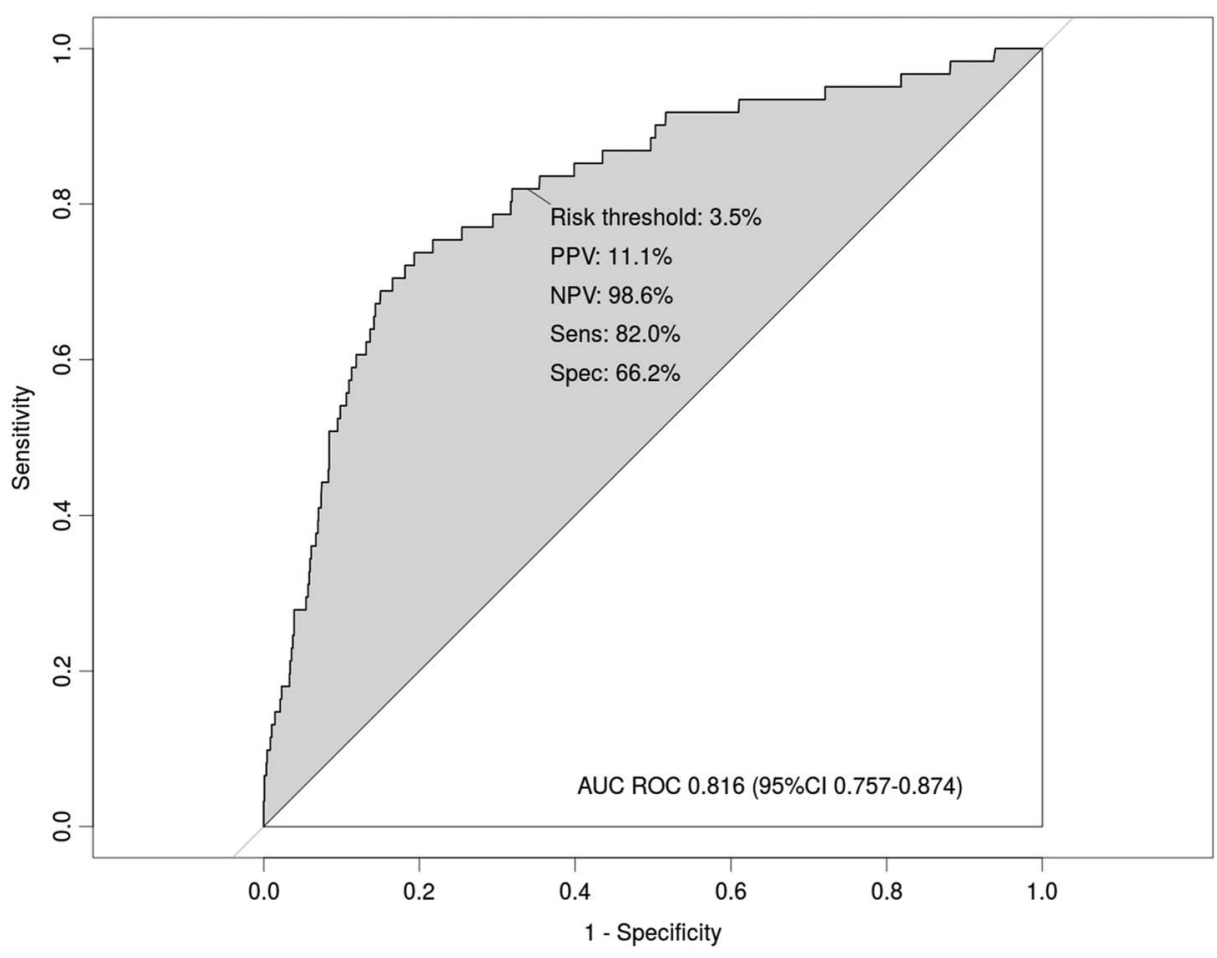


Table 4 Model characteristics at probability cut-offs ensuring model sensitivity of $>80 \%$

\begin{tabular}{lllllll}
\hline Model & AUC $(\mathbf{9 5} \% \mathbf{C l})$ & Probability cut-off & Sensitivity (95\% Cl) & Specificity (95\% Cl) & PPV & NPV \\
\hline 1 & $0.82(0.75$ to 0.87$)$ & 0.035 & $82.0(72.3$ to 91.6$)$ & $66.2(63.5$ to 68.9$)$ & 11.1 & 98.6 \\
2 & $0.81(0.75$ to 0.87$)$ & 0.040 & $80.3(70.4$ to 90.3$)$ & $67.5(64.8$ to 70.2$)$ & 11.3 & 98.5 \\
3 & $0.80(0.74$ to 0.86$)$ & 0.031 & $80.3(70.4$ to 90.3$)$ & $63.4(60.7$ to 66.2$)$ & 10.2 & 98.4 \\
4 & $0.80(0.73$ to 0.86$)$ & 0.035 & $82.0(72.3$ to 91.6$)$ & $61.4(58.6$ to 64.2$)$ & 9.9 & 98.5 \\
\hline \multicolumn{7}{l}{ AUC, area under the curve; NPV, negative predictive value; PPV, positive predictive value. }
\end{tabular}

inflammation and increased permeability) soon after birth can lead to early and irreversible stunting, frequent diarrhoeal illness and persistent systemic subclinical inflammation. ${ }^{23-26}$ These appear to promote a vicious cycle of infection and malnutrition. While difficult to address, a focus on nutrition (micronutrient and macronutrient) before, during and following the acute phase of illness may reduce the exacerbation of this cycle. Half of the children who died during the course of this study did so after >30 days following discharge. Therefore, emphasis must also be placed on preventing reinfection in vulnerable children. Promotion of good health behaviour (including hygiene) during the postdischarge period is, therefore, likely to play an important role.

One further area for intervention is education on timely health seeking. Sixty-seven per cent of the deaths in this study occurred outside of a hospital context, but $28 \%$ of the out-of hospital deaths occurred on the way to hospital. The education of mothers on the early warning signs of recurrent illness should also be emphasised during discharge since the common perception may be that recovery from infection brings a child back to a baseline level of risk, which is clearly not true. Since all children were enrolled during a hospital admission, physical inaccessibility was generally not an initial barrier. A previous study on the hospital burden of paediatric acute lower respiratory infections found that although $62 \%$ of children are treated in the hospital, $80 \%$ of deaths occur outside of the hospital. ${ }^{27}$ While this study did not address the timing of the out-of-hospital deaths in relation to the hospital visit, it is possible that many of these deaths occurred in the vulnerable months following discharge.

\section{CONCLUSION}

This study has derived a parsimonious risk-scoring tool for paediatric postdischarge mortality. Further work is required in external validation of this tool and for the development of effective postdischarge interventions.

\section{Author affiliations}

${ }^{1}$ School of Population and Public Health, University of British Columbia, Vancouver, British Columbia, Canada

${ }^{2}$ Department of Pediatrics, Mbarara University of Science and Technology, Mbarara, Uganda

${ }^{3}$ Center for International Child Health, BC Children's Hospital, Child and Family Research Institute, Vancouver, Canada

${ }^{4}$ Department of Pediatric Anesthesiology, BC Children's Hospital and University of British Columbia, Vancouver, Canada

${ }^{5}$ Canadian HIV Trials Network, St. Paul's Hospital and University of British Columbia, Vancouver, Canada
${ }^{6}$ Department of Pediatrics, BC Children's Hospital and University of British Columbia, Vancouver, Canada

${ }^{7}$ Maternal, Newborn and Child Health Institute, Mbarara University of Science and Technology, Mbarara, Uganda

${ }^{8}$ Department of Statistics, University of British Columbia, Vancouver, Canada

Twitter Follow Matthew Wiens at @matthew_wiens

Acknowledgements The authors gratefully acknowledge the contributions of Annet Twinomuguni, Justine Kamazima, Agaba Collins, Clare Komugisha, Solome Kobugyenyi, Alexander Mutungi and Hassan Bariahikwa. Without whose dedication, this study could not have been completed.

Contributors MOW, EK, CPL, JMA, JS, HW, AN, JKa, NK and JKi contributed to the study design. MOW, EK and JMA were responsible for the data collection. MOW, JS, HW and GZ carried out the date analysis. MOW contributed to the drafting of the initial manuscript. MOW, EK, CPL, JMA, JS, NK, HW, AN, JKa, GZ and JKi participated in the reviewing and revising of the manuscript. All the authors gave their approval for the final version of the manuscript.

Funding This study was internally funded. MOW was supported by a fellowship from the Canadian Institutes of Health Research, the Michael Smith Foundation for Health Research and the Canadian Child Health Clinician Scientist Program.

Competing interests None declared

Ethics approval University of British Columbia (Canada) and Mbarara University of Science and Technology (Uganda).

Provenance and peer review Not commissioned; externally peer reviewed.

Data sharing statement No additional data are available.

Open Access This is an Open Access article distributed in accordance with the Creative Commons Attribution Non Commercial (CC BY-NC 4.0) license, which permits others to distribute, remix, adapt, build upon this work noncommercially, and license their derivative works on different terms, provided the original work is properly cited and the use is non-commercial. See: http:// creativecommons.org/licenses/by-nc/4.0/

\section{REFERENCES}

1. Liu L, Oza S, Hogan D, et al. Global, regional, and national causes of child mortality in 2000-13, with projections to inform post-2015 priorities: an updated systematic analysis. Lancet 2015;385: 430-40.

2. Wang $\mathrm{H}$, Liddell $\mathrm{CA}$, Coates $\mathrm{MM}$, et al. Global, regional, and national levels of neonatal, infant, and under-5 mortality during 1990-2013: a systematic analysis for the Global Burden of Disease Study 2013. Lancet 2014;384:957-79.

3. Wiens MO, Pawluk S, Kissoon N. Pediatric post-discharge mortality in resource poor countries: a systematic review. PLOS ONE 2013;8: e66698.

4. Karlen W, Dumont G, Petersen C, et al. Human-centered phone oximeter interface design for the operating room: Pulse Oximeter interfaced to a mobile device for anesthesia monitoring in the developing world. Proceedings of the International Conference on Health Informatics. Rome, Italy: SciTePress, 2011:433-8.

5. The WHO Child Growth Standards [Internet]. 2006 (cited 1 Mar 2015). http://www.who.int/childgrowth/standards/en/ 
6. Fleming S, Thompson M, Stevens R, et al. Normal ranges of heart rate and respiratory rate in children from birth to 18 years of age: a systematic review of observational studies. Lancet 2011;377:1011-18.

7. National High Blood Pressure Education Program Working Group on High Blood Pressure in Children and Adolescents. The fourth report on the diagnosis, evaluation, and treatment of high blood pressure in children and adolescents. Pediatrics 2004:114:555-76.

8. National Implementation Guidelines for HIV Counselling and Testing in Uganda [Internet]. Kampala, Uganda, 2010. http://library.health. go.ug/publications

9. Harris PA, Taylor R, Thielke R, et al. Research electronic data capture (REDCap) - a metadata-driven methodology and workflow process for providing translational research informatics support. $J$ Biomed Inform 2009;42:377-81.

10. Wiens MO, Kissoon N, Kumbakumba E, et al. Selecting candidate predictor variables for the modelling of post-discharge mortality: a protocol development project. Africa Health Sciences. In press.

11. Hosmer DW, Lemeshow S. Goodness of fit tests for the multiple logistic regression model. Commun Stat 1980;9:1043-69.

12. Van Buuren S, Groothuis-Oudshoorn K. Multivariate imputation by chained equations. J Stat Softw 2011;45:1-67.

13. Stone M. An asymptotic equivalence of choice of model by cross-validation and Akaike's Criterion. J R Statist Soc 1977;39:44-7.

14. Shibata R. Bootstrap estimate of Kullback-Leibler information for model selection. Stat Sin 1997;7:375-94.

15. World Health Organization. Integrated Management of Childhood IIIness: distance learning course. Geneva: World Health Organization, 2014.

16. Nguyen DT, Leung KK, McIntyre L, et al. Does Integrated Management of Childhood Illness (IMCI) training improve the skills of health workers? A systematic review and meta-analysis. PLoS ONE 2013;8:e66030.

17. Lester RT, Ritvo P, Mills EJ, et al. Effects of a mobile phone short message service on antiretroviral treatment adherence in Kenya (WelTel Kenya1): a randomised trial. Lancet 2010;376: 1838-45.
18. Obasola OI, Mabawonku I, Lagunju I. A review of E-health interventions for maternal and child health in sub-Sahara Africa. Matern Child Health J 2015;19:1813-24.

19. Mitchell M, Hedt-Gauthier BL, Msellemu D, et al. Using electronic technology to improve clinical care-results from a before-after cluster trial to evaluate assessment and classification of sick children according to Integrated Management of Childhood Illness (IMCI) protocol in Tanzania. BMC Med Inform Decis Mak 2013;13:95

20. Moïsi JC, Gatakaa H, Berkley JA, et al. Excess child mortality after discharge from hospital in Kilifi, Kenya: a retrospective cohort analysis. Bull World Health Organ 2011;89:725-32.

21. Islam MA, Rahman MM, Mahalanabis D, et al. Death in a diarrhoeal cohort of infants and young children soon after discharge from hospital: risk factors and causes by verbal autopsy. J Trop Pediatr 1996;42:342-7.

22. West TE, Goetghebuer T, Milligan $\mathrm{P}$, et al. Long-term morbidity and mortality following hypoxaemic lower respiratory tract infection in Gambian children. Bull World Health Organ 1999;77: 144-8.

23. Prendergast A, Kelly P. Enteropathies in the developing world: neglected effects on global health. Am J Trop Med Hyg 2012;86:756-63.

24. Keusch GT, Denno DM, Black RE, et al. Environmental enteric dysfunction: pathogenesis, diagnosis, and clinical consequences. Clin Infect Dis 2014;59(Suppl 4):207-12.

25. Kosek M, Guerrant RL, Kang G, et al. Assessment of environmental enteropathy in the MAL-ED cohort study: theoretical and analytic framework. Clin Infect Dis 2014;59(Suppl 4):239-47.

26. Jones KD, Thitiri J, Ngari M, et al. Childhood malnutrition: toward an understanding of infections, inflammation, and antimicrobials. Food Nutr Bull 2014;35:64-70.

27. Nair H, Simões EA, Rudan I, et al. Global and regional burden of hospital admissions for severe acute lower respiratory infections in young children in 2010: a systematic analysis. Lancet 2013;381:1380-90. 